\title{
13
}

\section{Axiomatic Design for Products, Processes, and Systems}

\author{
Christopher A. Brown
}

\subsection{The Axioms and Engineering Design as a Scientific Discipline}

\subsubsection{The Axioms}

The axioms were developed in the late nineteen seventies by Prof. Nam P. Suh and his students at MIT. The project was supported by the US National Science Foundation. They studied design processes and the quality of design solutions.

They found that the best designs had two things in common. These became Suh's design axioms.

Axiom one: Maintain the independence of the functional elements. Axiom two: Minimize the information content.

C. A. Brown $(\bowtie)$

Department of Mechanical Engineering, Worcester Polytechnic Institute, Worcester, MA, USA

e-mail: brown@wpi.edu

(C) The Author(s) 2020 
When these axioms are applied properly, the resulting design solution is the best it can be for a given set of functional requirements and candidate design parameters (Suh 1990). Beyond the proper application of the axioms, the value, quality, and virtue of the design solution, rests on the formulation of functional requirements and the generation of candidate design parameters, which are discussed below.

These design axioms can apply to all kinds of problem solving. This includes a daily agenda and an arrangement of items on a desk, an online electric vehicle (Suh and Cho 2017), a mobile harbor (Lee and Park 2010; Park and Suh 2011) or software, and organizations (Suh 1998).

Note that the word "design" can refer to a representation of an object that has been designed, which is also a design solution. "Design" can also refer to a process during which the design solution is developed.

Newton's laws also apply to solving a wide variety of problems, from planetary motion to apples falling on earth. Suh's axioms, like Newton's laws, and any other set of axioms or natural laws, cannot be proven. Their validity relies on the lack of observations to the contrary within their domains of applicability.

\subsubsection{Three Parts and Six Elements}

Axiomatic design has three essential parts: axioms, structure, and process. Each of these three parts can be decomposed into two elements, as shown in Table 13.1.

The axioms are applied during the design process. Design problems are formulated and design solutions developed, from abstract to detailed, down hierarchies of abstraction and across functional, physical and process domains, during a zigzagging decomposition process, so that the design solution is consistent with the axioms.

\subsubsection{Axiom One: Maintain Independence}

Adherence to the independence axiom assures that design solutions avoid unintended consequences, and that it will be adjustable and controllable. It does this by developing a design solution that avoids 
Table 13.1 Parts and elements of design

\begin{tabular}{|c|c|c|}
\hline Parts & Elements & Notes \\
\hline \multirow[t]{2}{*}{ 1. Axioms } & $\begin{array}{l}\text { 1.1 Maintain the independence } \\
\text { of the functional elements }\end{array}$ & $\begin{array}{l}\text { Facilitates adjustment, } \\
\text { controllability, and avoids } \\
\text { iteration and unintended } \\
\text { consequences }\end{array}$ \\
\hline & $\begin{array}{l}\text { 1.2 Minimize the information } \\
\text { content }\end{array}$ & $\begin{array}{l}\text { Maximizes probability (p) of } \\
\text { success. } I=\ln (1 / p)\end{array}$ \\
\hline \multirow[t]{2}{*}{ 2. Structures } & 2.1 Design domains & $\begin{array}{l}\text { Decomposes laterally by type } \\
\text { of domains, customer, func- } \\
\text { tional, physical, and process }\end{array}$ \\
\hline & 2.2 Design hierarchies & $\begin{array}{l}\text { Decomposes vertically by level } \\
\text { of detail in the domains }\end{array}$ \\
\hline \multirow[t]{2}{*}{ 3. Processes } & 3.1 Zigzagging decomposition & $\begin{array}{l}\text { Developing detail down } \\
\text { through the hierarchical } \\
\text { domains }\end{array}$ \\
\hline & 3.2 Physical integration & $\begin{array}{l}\text { Integrating detailed physical } \\
\text { components up through the } \\
\text { physical domain }\end{array}$ \\
\hline
\end{tabular}

unwanted coupling. When two functions are coupled, they cannot be adjusted or changed independently, i.e., a change in one influences the other, to bring about a change in it too. The result is that some non-productive iteration will generally be required to reach the desired result, if indeed a convergent adjustment strategy can be found. Coupling can occur when one physical component is used to satisfy two FRs. This frequently occurs as the result of well-meaning attempts to reduce the number of components, believing that this will save cost and simplify the design. The result can be just the opposite.

\subsubsection{Axiom Two: Minimize the Information Content}

Information content is the log of one divided by the probability of success $I=\ln (1 / p)$ Information content in design in this sense is not related to knowledge. The first axiom can be seen to be a special case of the first, because the independence promotes success. Axiom two is applied after the first. It is used to select between candidate solutions, favoring those with the greatest possibility of fulfilling the functions and satisfying the customer needs. 


\subsection{Structures}

In order to apply the axioms, the developing design solution should be structured so unwanted coupling and information content are obvious and can be designed out. The need to apply the axioms to the developing design solution, during the design process, drives the design structure. The form of the design process is based on the functions required for the process of conceptualizing and developing design solutions. The axiomatic design process is therefore based on the axioms and is thereby self-consistent.

The completed design solution structure is a verbal description of the design solution. Its construction is essential for completing the design solution. The design structure is like a free body diagram for the design problem, a multidimensional, orthogonal description of the functions, and solutions.

The structure consists of design domains laterally, and hierarchies of detail within the domains, vertically. The design domains are different kinds of descriptions of the design. The functional requirements (FRs), items in the functional domain, are formulated to satisfy the Customer Needs (CNs), items in the customer domain. FRs are fulfilled by the physical solutions, i.e., the design parameters (DPs), which are items in the physical domain. The DPs are created with Process Variables (PVs), which are items in the process domain.

The design domains are listed in Table 13.2, along with the elements of which they consist, their abbreviations, and their descriptions. The items in the different design domains connect to each other.

Table 13.2 The design domains

\begin{tabular}{|c|c|c|c|c|}
\hline Domains & Customer & Functional & Physical & Process \\
\hline Items & $\begin{array}{l}\text { Customer } \\
\text { Needs }\end{array}$ & $\begin{array}{l}\text { Functional } \\
\text { Requirements }\end{array}$ & $\begin{array}{l}\text { Design } \\
\text { Parameters }\end{array}$ & $\begin{array}{l}\text { Process } \\
\text { Variables }\end{array}$ \\
\hline Abbreviations & CNs & FRs & DPs & PVs \\
\hline $\begin{array}{l}\text { Grammatical } \\
\text { form }\end{array}$ & Anything & $\begin{array}{l}\text { Imperative: begins } \\
\text { with a verb }\end{array}$ & $\begin{array}{l}\text { Adjectives and } \\
\text { nouns }\end{array}$ & $\begin{array}{l}\text { Present } \\
\text { participles }\end{array}$ \\
\hline Notes & $\begin{array}{l}\text { What is } \\
\text { needed }\end{array}$ & What it does & How it looks & $\begin{array}{l}\text { How to pro- } \\
\text { duce it }\end{array}$ \\
\hline Other & & Constraints Cs & & \\
\hline
\end{tabular}




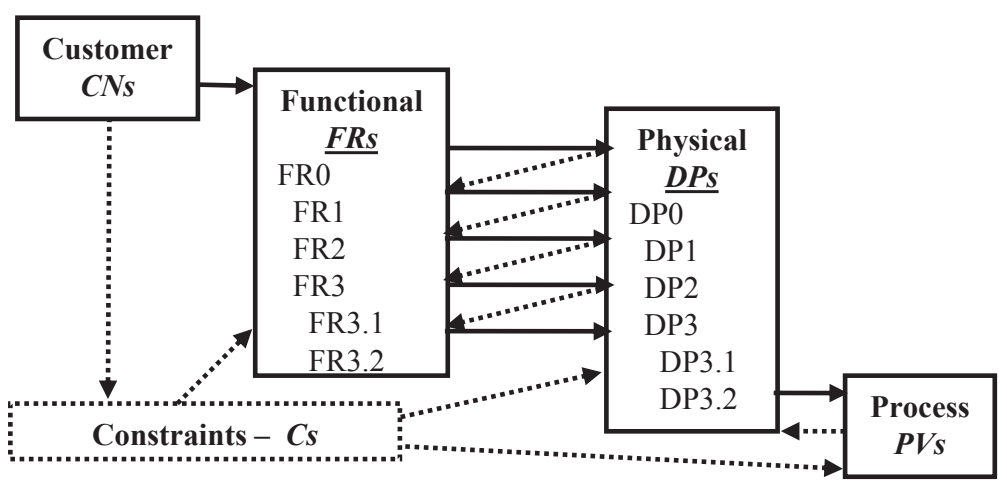

Fig. 13.1 Design domains, customer, functional, physical, and process, and the constraints, with their components. Zigzagging decomposition is indicated between the functional and physical domains. Upper level DPs constrain lower level FRs. The process domain and its connection to the physical domain is not elaborated here

These connections are evaluated by the axioms, particularly axiom one. The CNs describe what will be valued in the design. There is a value chain that runs from the Customer Domain to the Functional Domain, to the Physical Domain, and then on to the Process Domain (Fig. 13.1).

\subsubsection{Design Domains and Constraints-Lateral Decompositions}

The design domains (see Table 13.2) are spaces, or zones, on a field where a design game can be played. When the design decomposition is complete here is should be one-to-one correspondences between the items in the functional, physical, and process domains. Each FR needs its own DP, which should have a corresponding PV.

\subsubsection{Customer Needs (CNs)}

The Customer Domain describes what customers want, i.e., what they would value. Thompson (2013a) provides an excellent description of the process of identifying the customer and other stakeholders, 
e.g., manufacturing, transport, and salespeople. CNs might also contain constraints (Cs), things that must be avoided, and preferences that can become selection criteria (SCs) or optimization criteria (OCs) as described by Thompson (2013b).

In practice, $\mathrm{CNs}$ could be developed by a marketing group. Describing CNs can begin a design process. CNs have no special grammatical form. They should describe the fundamental needs, preferences, and constraints in a way that opens the solution space appropriately.

\subsubsection{Functional Requirements (FRs)}

The FRs should be an organized technical description of what the design must do, i.e., the functions it needs to provide. FRs are stated in the imperative. They begin with verbs. At the highest levels, FRs can be things like "transport people," or "deliver hot water."

Most often just the top-level FRs are developed from the CNs, because the CNs tend to be more abstract. The initial FRs should be the minimum list of functions that satisfy the CNs. The FRs should be collectively exhaustive with respect to the CNs. At any level, FRs should be mutually exclusive with respect to each other. Formulating good FRs is essential. A design solution cannot be better than its FRs. The top-level FRs establish the starting point, indicate the direction, and define when the destination is reached.

The FRs should state the design objective directly, for example, "transport people." A common mistake of novices is to make "design a bicycle" an FR, intending to design a new kind of bicycle. The operative verb is "design," so this suggests designing a system for designing a bicycle. If the intent is to design a new sort of bicycle then the FR might rather be "transport people." It could have constraints to be powered by the people being transported. Using a term like "bicycle" already suggests a physical solution, thereby limiting the solution space, and reducing the potential for a new, creative solution to the transport problem. 


\subsubsection{Design Parameters (DPs)}

DPs describe what the design looks like. What eventually appears in a CAD file for mechanical parts, or lines of code for software. DPs begin with nouns. At the highest levels, DPs are things like "personal transportation device" or "hot water system." At the lower levels DPs can be things like "ball bearings" or "valves," or the design can start there, if you are designing rolling elements or fluid control devices.

The DPs are the physical items that fulfill the FRs. In order to comply with axiom one, the independence axiom, in the completed decomposition there should be one and only one DP corresponding to each FR. Ideally, that DP should only influence the FR that it is intended to fulfill.

The term "physical," referring to the physical domain and physical attributes, is not necessarily physical literally. DPs can also be "computer code," "education modules," or "slots of time in an agenda." These DPs would fulfill FRs like "calculate best fit line," "teach engineering design," or "meet with clients."

\subsubsection{Process Variables (PVs)}

PVs describe how the DPs are produced. PVs can be manufacturing processes, like machining, injection molding, and assembly. The PVs can be developed so that the PVs have a one-to-one relation with the DPs. This can be necessary when functional and physical tolerances are difficult to achieve (Brown 2018). Often it is not necessary to develop this kind of detail in the Process Domain. When it is time to do process design, then it can be advisable to begin a new design problem with FRs and DPs relating to the process (Brown 2014).

\subsubsection{Constraints (Cs)}

Cs interface with the domains, although they are not in the value chain. Cs are limits or restrictions on the items in the other domains. Cs sometimes cannot be decoupled from the other items in the domains. 
They are things that must be avoided rather than fulfilled. Cs differ from FRs in that Cs do not have DPs. Another important difference is that while each of the FRs should be independent of all other FRs, Cs can influence all the FRs and DPs, e.g., weight and cost limitations.

\subsubsection{Design Hierarchies-Vertical Decomposition}

When more than one level of detail is necessary to describe a design solution to the point where the solution is obvious, then the design should be decomposed vertically. This decomposition can continue increasing in detail, from parent to child, through several generations, or levels, until the solution is obvious. The upper level FRs describe main branches, subsequent decomposition of the children define branches that are more detailed. A decimal notation is used for the branches. The top-level FR is FR0. The next level are FR1, FR2, FR3, etc. The children of FR1 are FR1.1, FR1.2, FR1.3, etc.

\subsection{The Design Process}

The design process seeks first to generate items comprising a design solution by systematically decomposing abstract design concepts into progressively more detailed items until the decomposed solution is obvious. Then the design decomposition is physically assembled to produce a complete, integrated solution. The decomposition and integration are both done so as to assure that the resulting design solution complies with the axioms. A complete solution should include functional and physical metrics with dimensioning and tolerancing. Steps in the design process are shown in Table 13.3.

\subsubsection{Zigzagging Decomposition}

The decomposition is generated by zigzagging between domains at one level, then proceeding to the next, more detailed level (Fig. 13.2). Generally, this is done from the functional to the physical domain, 
Table 13.3 Steps for zigzagging decompositions to develop FRs and select the best DPs

1. Identify the needs of the customers and stakeholders (CNs) (Thompson 2013a)

2. Develop FRO based on CNs

2.1 Select DPO candidates

2.2 Select best DPO based on minimizing information content (Axiom 2)

3. Develop next level or branch of FRs, based on CNs where appropriate

3.1 Check for non-FRs, OCs, SCs (Thompson 2013b) and violation of constraints

3.2 Check FRs for CEME decomposition (Axiom 1), and correct if necessary

4. Select corresponding DP candidates for each FR, and check for violation of constraints

5. Select the best DP for each FR from the candidates

5.1 Select those that maintain independence (Axiom 1-use design matrix)

5.2 Select best for minimum information content (Axiom 2 - use $I=\log (1 / p)$ )

6. If the solution is obvious, then leave the decomposition and go to physical integration, if not, go to step 3

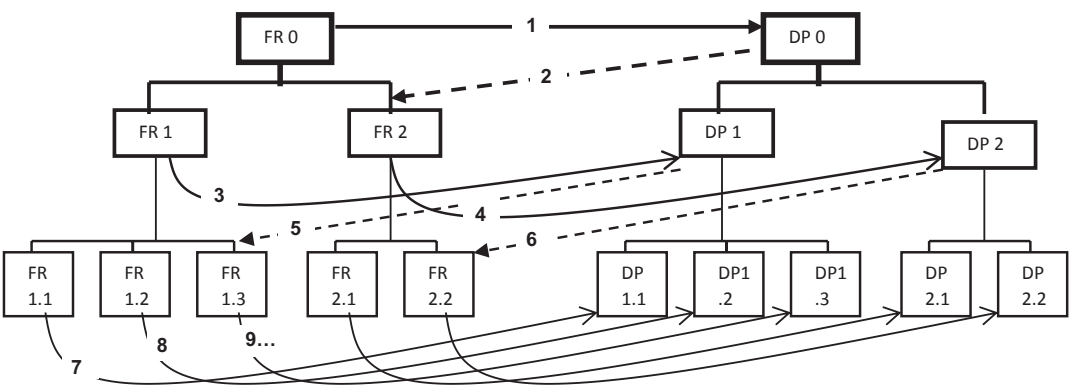

Fig. 13.2 Zigzagging decomposition symbolic example. The arrows show the direction of the flow and the order is indicated by the numbers on the arrows. There must be at least two children at each level of the decomposition in each branch

although it can be extended to the process domain as well. Each parent must have at least two children in its domain.

The children contain more detail than the parent does. The children should combine to equal the parent, that is, they must be collectively exhaustive (CE) with respect to the parent. If not, the decomposition is 
incomplete. The children at each level in each branch must be mutually exclusive (ME) with respect to each other, in order to comply with axiom one, independence. An appropriate decomposition is said to be CEMEmin, indicating in addition the minimum number of FRs.

In these hierarchal decompositions upper level DPs constrain lower level FRs. If, for example, a pneumatic solution has already been selected at a higher level, then all subsequent lower-level solutions should fit with the higher-level choice, for example, pneumatic valves and actuators.

\subsubsection{Checking for Independence (Axiom One)}

At each level and on each branch of the decomposition the proposed solutions should be checked for compliance with the axioms. This is done by examining the design matrix, which, minimally, shows which DPs influence which FRs (Tables 13.4, 13.4, 13.5 and 13.6).

Table 13.4 Uncoupled basic design matrix, diagonal, and therefore fully independent

\begin{tabular}{llll}
\hline & DP1 & DP2 & DP3 \\
\hline FR1 & $X$ & 0 & 0 \\
FR2 & 0 & $X$ & 0 \\
FR3 & 0 & 0 & $X$ \\
\hline
\end{tabular}

Table 13.5 Decoupled basic design matrix, triangular, and therefore quasi-independent

\begin{tabular}{llll}
\hline & DP1 & DP2 & DP3 \\
\hline FR1 & $X$ & 0 & 0 \\
FR2 & $X$ & $X$ & 0 \\
FR3 & $X$ & $X$ & $X$ \\
\hline
\end{tabular}

Table 13.6 Coupled basic design matrix, full, and therefore not independent

\begin{tabular}{llll}
\hline & DP1 & DP2 & DP3 \\
\hline FR1 & $X$ & $X$ & $X$ \\
FR2 & $X$ & $X$ & $X$ \\
FR3 & $X$ & $X$ & $X$ \\
\hline
\end{tabular}


The Xs in the matrices indicate that the DP in the upper row influences the FR in the left column. The nature of the relation could be described in any way. The exact nature of the relation need not be known, only that an imaginable change in the DP could be enough to take the FR out of tolerance.

An uncoupled design matrix is diagonal. Each DP influences only one FR. The DPs can be adjusted in any order to satisfy all FRs without any non-productive iterations. This is the best.

A decoupled design matrix has one or more off-diagonal interactions on the same side of the diagonal (Table 13.5). There are certain orders for adjusting DPs that can fulfill the FRs without non-productive iterations. In the case of a fully triangular matrix there is only one order of adjustment of the DPs to fulfill the FRs. In Table 13.5, which is lower triangular, the adjustment order is $1,2,3$. The order of adjustment for an upper triangular matrix would be $3,2,1$. Some matrices, which are not triangular, can be rearranged to be triangular using linear algebra. Triangular is acceptable. Favor those solutions with the most offdiagonal zeros in their design matrices.

A coupled matrix (Table 13.6) has at least one interaction (X) on both sides of the diagonal and no way to rearrange to the matrix to triangular. There is no order of adjustment without iteration. Even with iteration, there is no guarantee of convergence on an acceptable solution. This matrix indicates a failure to be adjustable, controllable, and to avoid unintended consequences. Another solution should be sought with more independence.

\subsubsection{Decomposition Themes}

Themes can be used to facilitate CEME decompositions. Different themes can be used at different levels. Energy, work, and partition, by location or by sequences in time, are useful themes in manufacturing. In testing for being $\mathrm{CE}$, each form of energy and work, location or instance of time can be accounted for to insure the decomposition is exhaustive. For example, in response to a $\mathrm{CN}$ to improve sustainability in manufacturing an FR might be to recover waste in machining. One branch might be to recover thermal energy. This can be partitioned into 
Table 13.7 Example of an FR-DP, top-level decomposition in response to a CN for sustainability in metal cutting

\begin{tabular}{ll}
\hline FR0 recover waste heat in machining & DP0 heat recovery system \\
\hline FR1 Recover heat from the tool & DP1 Thermoelectric generator \\
FR2 Recover heat from the chips & DP2 Gaseous heat exchanger in chip \\
& handler \\
FR3 Recover from the workpiece & DP3 Liquid heat exchanger with cut- \\
& ting fluid \\
\hline
\end{tabular}

locations that contain excess thermal energy as the result of the work during machining, the chips, the tool, and the workpiece (Table 13.7).

Location specific heat recovery systems could be selected as corresponding DPs. Selected themes can influence the process, as well as the ability to apply the axioms. Different themes, or themes selected in a different sequence at different levels, can result in the same elemental items in the decomposition at the lowest levels. An alternative decomposition could be made by the temperature of the heat, which would have had to have been addressed eventually in a decomposition beginning with a location theme, because of the fundamental thermodynamics of heat recovery.

Another theme for decomposition can be directional, e.g., Cartesian. For example, limit or control movement could be decomposed into controlling or limiting movement in three orthogonal directions. Another kind of theme can be provided by terms used in the FRs. The FRs are at least a verb-noun pair. Often it is possible to decompose the verb and the noun into two or more components each.

\subsubsection{Metrics and Equations}

Metrics and equations can assist with the decomposition process, although frequently the decomposition is done qualitatively, at least initially. The FRs benefit from functional metrics that indicate the extent to which the function is satisfying a CN. The DPs need physical metrics, which can be measures of physical properties. The PVs need process metrics, which are adjustments in the process domain. 
Two kinds of equations can be useful in the decomposition. Equations relating two different domains. The FRs and DPs are related by the design equations (13.1). The DPs and the PVs are related by the process equations (13.2).

$$
\begin{aligned}
& F R i=f(D P j) \\
& D P j=g(P V k)
\end{aligned}
$$

The functions $\mathrm{f}$ and $\mathrm{g}$ can be described in detailed design and process matrices. The FRs, DPs, and PVs can be described as column vectors. When written in differential form, which is useful because the interest is in how changes in the DPs change the FRs, the design and process matrices are the Jacobians, describing these changes.

Equations can be used to relate the parents and the children. These could be used to verify that the decomposition is CEME.

$$
\mathrm{FRi}=\text { FRi. } 1+\text { FRi. } 2 \text { + FRi. } 3 \ldots
$$

They do not have to be related by a summation, although this is the simplest relation. Any kind of combination of the children to achieve the parent would suffice.

\subsubsection{Physical Integration}

When the decomposition is complete, all the physical items for the design have been selected. Then for a complete solution, the physical items need to be physically integrated. This could be a drawing or some other representation of the juxtaposition of the physical parts of the design solution. Note that physical integration can be in space or time.

The branches and themes in the physical integration can differ from those in the decomposition. Some items need to be proximate to provide the intended function. In a car, for example, the braking system could be one branch in the decomposition and be dispersed in the physical structure of the car after the physical integration.

It is good practice to include the FR-DP numbers in the representation, so each physical item (DP) can be traced back to an FRs in the 
decomposition and the intent can be identified. In this way, the consequences of modifications can be quickly traced and unintended consequences avoided.

To facilitate the physical integration, a DP-DP matrix or a PV-PV matrix can be created. It can show the interactions, or lack of them, with $\mathrm{Xs}$ and 0 s, like the design or process matrices. The diagonal is superfluous, because it indicates that an item interacts with itself. The off-diagonal Xs indicate physical interactions, in space or time that are introduced by the integration. These need to be checked to be sure the independence is not compromised (Axiom 1) and that the information is not increased unnecessarily beyond some equally functional physical configurations (Axiom 2).

\subsection{Additional Applications and Industry 4.0}

This section and the following subsections discuss some applications and aspects of $\mathrm{AD}$ that could be of particular interest to people doing work related to Industry 4.0 (I4.0).

As we look to integrate Industry 4.0 and $\mathrm{AD}$, we note that the current descriptions of Industry 4.0 seem to fit best as items in the physical domain, as DPs. Industry 4.0 seems to comprise things like cyber-physical production systems (CPPS), connectivity for data exchange and automated decision-making for routine situations. Industry emphasizes solutions in the physical domain, i.e., DPs. The task then is understanding the appropriate functions, FRs, and the CNs that would use these DPs. In other words, engineers should decide how these interesting tools should be used ethically for the benefit of humanity.

\subsubsection{Analyzing Existing Designs}

The AD method as described above is intended to be used on new design problems. It can be difficult or impossible to fix an existing design to make it adjustable, controllable, robust, and avoiding unintended consequences. Nonetheless, there are techniques that can be used to study and improve existing designs. 
Design thinking starts with knowing or formulating the intended functions, i.e., the design intent. Often problems result from not adequately considering and articulating the functions. The functions must satisfy needs defined by the customers and stakeholders. Improving an existing design solution or portion of it, by using $\mathrm{AD}$, generally requires doing a decomposition, for at least of some part of the design solution.

Problems can also arrive in achieving a solution when a design effort has been partitioned too early in the design process. This can result from an assumption that independence of branches can be maintained during subsequent hierarchal decomposition and integration of these branches. The solutions in some branches can be more challenging than in others, and these branches might need a higher priority in allocation of constraints, such as cost, space, and weight. This assumes that it will be easier to adapt the less challenging branches to comply with system-wide constraints.

Many poor designs can be linked to specific problems (Table 13.8). These problems can be related to failing some aspect of the axioms, which can be linked to shortcomings in the process. References in Table 13.8 suggest some solutions.

In some cases, new CNs are introduced, as with items 1 and 2 in Table 13.8. This requires the formulation of new FRs, and maybe $\mathrm{nFRs}$ (Cs, OCs, and SCs, Thompson 2013a), and returning to procedures in Table 13.3.

Items 3, 4, and 5 in Table 13.8 require the selection of new DPs and returning to procedures in Table 13.3. Sometimes, if there are

Table 13.8 Some typical problems in a poor design

1. Poor understanding of the CNs and stakeholder needs (Thompson 2013a)

2. Not developing appropriate FRs and nFRs (Thompson 2013b)

3. Having fewer DPs than the FRs (violates axiom one)

4. Selecting DPs that influence too many FRs (violates Axiom one)

5. Difficulties achieving solutions consistently (low yield, violates Axiom two)

6. Not knowing the correct order of adjustment (imaginary complexity according to Suh 2005)

7. Violation of constraints (Axiom two)

8. Poor physical integration introduces coupling (Axiom one), or reduces probabilities of success (Axiom two) 
difficulties finding DPs, then the FRs might need modifications to enlarge the solution space as described in Sect. 13.4.2 below.

Imaginary complexity (item 6 in Table 13.8) can be addressed by constructing a design matrix and decoupling using linear algebra to show the correct order of adjustment (Suh 1990).

There are times when the existing design solutions should be modified to reduce cost, weight, or volume, a constraint violation (item 7 Table 13.8). An approach is to develop the decomposition. Starting with the CNs, including all the stakeholders, clearly establish what provides the required value. Look at each item in the solution FRs, DPs, and PVs, moving through the value chain and identify those things that add cost, weight, or volume and do not add value, and then eliminate them. Then examine DPs and PVs that add to the constrained quantities, i.e., Cs, and look for alternative solutions that add less and still comply appropriately with the axioms.

Item 8 in Table 13.8 suggests a return to the physical integration procedure, and an examination of the physical integration matrices (DP-DP, or PV-PV). If the necessary independence or information content cannot be achieved, then this indicates a return to the FRs. Perhaps some FRs need to be modified to increase the solution space (Sect. 13.4.2), while still satisfying the CNs, and new DPs or PVs found. Maybe the CNs need to be better understood (see Sect. 13.4.2).

\subsubsection{Recognizing Opportunities for Creativity and Innovation}

Being creative is having good, useful ideas. Innovation is making them viable. The decomposition and integration processes are a kind of functional modeling. These can help to make a good idea viable.

Working in the best solution space is important for creativity. FRs need to be solution neutral. This starts with understanding the CNs to formulate good FRs.

Henry Ford supposedly said that if he had asked people what they wanted, they would have said a faster horse. The issue is then to understand that the desire was for faster transportation. There is a tendency 
to go with known solutions, like a horse for transportation at the beginning of the twentieth century.

The FRs should not limit the DPs unduly. One test for a good FR is to see how many candidates DPs can fit with the FR. Be skeptical of FRs that do not provide enough solution space for several candidate DPs. In these cases check to see if the FR suggests a physical solution. Sometimes, when the FR is too close to the DPs, the FR can be moved closer to the $\mathrm{CN}$ and provide more solution space for the DPs.

Another approach is to ask why that FR is needed. Often in the answer to this question is another FR that will also satisfy the same $\mathrm{CNs}$, although in a more fundamental way that provides a larger solution space for selecting candidate DPs.

\subsubsection{Application for Industry 4.0}

For enterprises, a good $\mathrm{CN}$ and FR0 can be a return on investment (ROI). This suggests a decomposition for based on return and on investment (Suh 2001). It can be tempting to make the next level FRs maximize return and minimize investment. Maximizing and minimizing only make good FRs when there is a system or program for achieving the maximum or minimum, which could be a DP.

The question for I4.0 might be, do the DP heavy descriptions fit into solutions for ROI. The temptation to use a I4.0 type solution without looking to see if there is another solution that works and requires less investment. The decision to use a I4.0 type solution should be justified in terms of ROI, and in terms of satisfying the axioms.

New technologies can also be used to fulfill FRs that could not previously be considered. It is in this vein that the best use might be made of the I4.0 type solutions. In the pursuit of Industry 4.0 there are $\mathrm{CNs}$ and resulting FRs that should be considered now, which were not previously, because there appeared to be no physical solution. Industry 4.0 can enable new design solutions for production systems that were previously unattainable. These new design solutions might help to reverse climate change, and provide appropriate ROIs, through improved productivity. 


\subsection{Concluding Remarks}

Considering the CNs, for all the stakeholders in Industry 4.0, engineers must hold paramount the safety, health, and welfare of the public (first fundamental canon of ethics for engineers). Engineers must act according to the canons of ethics, otherwise what they are doing is not engineering. Industry 4.0 must consider sustainability because it is essential to the safety, health, and welfare of the public.

In 1953, a few years after the invention of the transistor as the first digital large computers appeared Kurt Vonnegut published Player Piano (Vonnegut 1953). The title appears to a reference to John Parson's first numerical controls for machine tools with punch tapes and pneumatic actuators. This novel describes a highly automated, yet highly disturbing, society, which is in the grips of another industrial revolution with some similarities to Industry 4.0. It mentions that this later industrial revolution devalues human thought, just as the first industrial revolution devalued human labor.

Axiomatic Design is powerful. It can empower tools that are available and being developed for Industry 4.0. AD and Industry 4.0 must be used for the benefit of humanity. Sustainability and the preservation and enhancement of freedom and dignity, must be included in the CNs. If Industry 4.0 diminishes these things, and serves mainly to enrich further those who are already wealthy, then it has failed.

\section{References}

Brown, C.A. 2014. Axiomatic Design of Manufacturing Processes Considering Coupling. In Proceedings of the 8th International Conference on Axiomatic Design, 149-153, Sept 24-26, Campus de Caparica, Portugal.

Brown, C.A. 2018. Specification of Surface Roughness Using Axiomatic Design and Multiscale Surface Metrology. Procedia CIRP 70: 7-12. https:// doi.org/10.1016/j.procir.2018.03.094.

Lee, T., and G.J. Park. 2010. Managing System Design Process Using Axiomatic Design: A Case on KAIST Mobile Harbor Project. SAE International Journal of Materials and Manufacturing 3 (1): 125-132. https://doi.org/10.4271/2010-01-0278. 
Park, N.K., and S.C. Suh. 2011. Modal Shifting from Road to Coastal Shipping Using a Mobile Harbor. The Asian Journal of Shipping and Logistics 27 (3): 447-462. https://doi.org/10.1016/S2092-5212(11)80021-0.

Suh, N.P. 1990. The Principles of Design. New York: Oxford University Press. Suh, N.P. 1998. Axiomatic Design Theory for Systems. Research in Engineering Design 10 (4): 189-209. https://doi.org/10.1007/s001639870001.

Suh, N.P. 2001. Axiomatic Design: Advances and Applications. MIT-Pappalardo Series in Mechanical Engineering. New York: Oxford University Press.

Suh, N.P. 2005. Complexity: Theory and Applications. New York: Oxford University Press.

Suh, N.P., and D.H. Cho. 2017. The On-Line Electric Vehicle: Wireless Electric Ground Transportation Systems. Cham: Springer.

Thompson, M.K. 2013a. Improving the Requirements Process in Axiomatic Design Theory. CIRP Annals 62 (1): 115-118. https://doi.org/10.1016/j. cirp.2013.03.114.

Thompson, M.K. 2013b. A Classification of Procedural Errors in the Definition of Functional Requirements in Axiomatic Design Theory. In Proceedings of the 7th International Conference on Axiomatic Design, June 27-28, Worcester, MA, USA.

Vonnegut, K. 1953. Player Piano: A Novel. London: Macmillan.

Open Access This chapter is licensed under the terms of the Creative Commons Attribution 4.0 International License (http://creativecommons. org/licenses/by/4.0/), which permits use, sharing, adaptation, distribution and reproduction in any medium or format, as long as you give appropriate credit to the original author(s) and the source, provide a link to the Creative Commons license and indicate if changes were made.

The images or other third party material in this chapter are included in the chapter's Creative Commons license, unless indicated otherwise in a credit line to the material. If material is not included in the chapter's Creative Commons license and your intended use is not permitted by statutory regulation or exceeds the permitted use, you will need to obtain permission directly from the copyright holder.

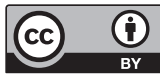

